\title{
Conductive Black Titania Nanomaterials for Efficient Photocatalytic Degradation of Organic Pollutants
}

\author{
Qingyuan $\mathrm{Bi}^{1} \cdot$ Xieyi Huang ${ }^{1} \cdot$ Yanchun Dong ${ }^{2} \cdot$ Fuqiang Huang ${ }^{1,3}$
}

Received: 23 July 2019 / Accepted: 26 August 2019 / Published online: 25 November 2019

(C) The Author(s) 2019

\begin{abstract}
Titanium dioxide $\left(\mathrm{TiO}_{2}\right)$ as an important semiconductor is widely used in the fields of solar cell, solar thermal collectors, and photocatalysis, but the visible-light power harvest remains insufficient due to the little effective visible-light absorption and many carrier-recombination centers originating from the wide band gap structure. Herein, conductive black titania (BT) nanomaterials with crystalline- $\mathrm{TiO}_{2}$-core/amorphous- $\mathrm{TiO}_{2-\mathrm{x}}$-shell structure prepared through two-zone Al-reduction route are found efficient in photocatalyzing the degradation of organic pollutants to environmentally friendly products under full solar and even visible light irradiation. The unique core-shell structure and numerous surface oxygen vacancies or $\mathrm{Ti}^{3+}$ species in the amorphous layer accompanying prominent physicochemical properties of narrow band gap, high carrier concentration, high electron mobility, and excellent separation and transportation of photoinduced $\mathrm{e}^{-}-\mathrm{h}^{+}$pairs result in exceptional photocatalytic efficiency. The optimized BT-500 (pristine $\mathrm{TiO}_{2}$ treated at $500{ }^{\circ} \mathrm{C}$ during two-zone Al-reduction process) catalyst achieves superior photocatalytic degradation rates for toluene and ethyl acetate as well as an excellent photostability with high degradation efficiency of $93 \%$ for the 6th reuse.
\end{abstract}

\section{Graphic Abstract}

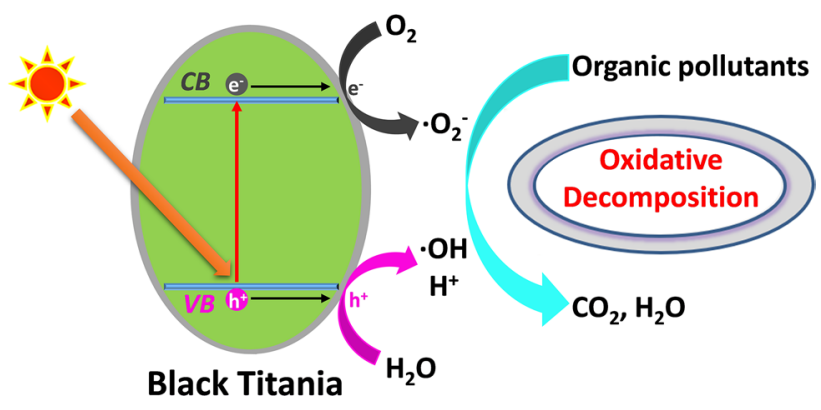

Keywords Black titania $\cdot$ Crystalline-core/amorphous-shell structure · Oxygen vacancies · Organic pollutants · Photocatalytic degradation

Electronic supplementary material The online version of this article (https://doi.org/10.1007/s10562-019-02941-1) contains supplementary material, which is available to authorized users.

Yanchun Dong

dycfsh00@sina.com

Fuqiang Huang huangfq@mail.sic.ac.cn

1 State Key Laboratory of High Performance Ceramics and Superfine Microstructure, Shanghai Institute of Ceramics, Chinese Academy of Sciences, Shanghai 200050, People's Republic of China
2 State Key Laboratory of NBC Protection for Civilian, Institute of Chemical Defense, Chinese Academy of Military Sciences, Beijing 100191, People's Republic of China

3 State Key Laboratory of Rare Earth Materials Chemistry and Applications, College of Chemistry and Molecular Engineering, Peking University, Beijing 100871, People's Republic of China 


\section{Introduction}

Toxic organic pollutants, like hydrocarbons, esters, aldehydes, and phenols, emitted from chemical industries and modern life can lead to serious environmental pollution and health issues [1,2]. Great efforts have been made to reduce the emission of organic pollutants, as well as their effective collection and degradation. Photocatalytic degradation of organic pollutants via catalytic oxidation process possesses two advantages of (i) renewable and clean solar energy as energy sources and (ii) the reaction products of $\mathrm{CO}_{2}$ and $\mathrm{H}_{2} \mathrm{O}$ showing no secondary pollution [3-5]. Therefore, the photocatalytic degradation strategy is considered as a promising and economic approach to resolve the toxic organic pollution. Efficient photocatalytic degradation of organic pollutants requires excellent photocatalyst to break the strong chemical bonds (e.g., $\mathrm{C}-\mathrm{C}, \mathrm{C}-\mathrm{H}$, and $\mathrm{C}=\mathrm{O}$ bonds) of organic molecule [6]. The commonly used catalysts are indispensable active components of noble metals (likely $\mathrm{Pd}, \mathrm{Pt}, \mathrm{Au}$, and $\mathrm{Ag}$ ) coupled with semiconductors [7-9]. Exploration of alternative cost-efficient materials is also necessary to replace the noble catalysts. Titanium dioxide $\left(\mathrm{TiO}_{2}\right)$ as the wellknown photo(electro)catalyst is widely used in the fields of water splitting, methyl orange degradation, $\mathrm{CO}_{2}$ reduction, and so on [10-15]. However, the efficiencies of fullspectrum solar utilization are still very low because of the large band gap of 3.0-3.2 eV, poor visible (Vis) and even infrared (IR) light response, low carrier concentrations, weak separation and transportation of photoinduced electron-hole $\left(\mathrm{e}^{-}-\mathrm{h}^{+}\right)$pairs [16]. Therefore, the microstructures and physicochemical properties of the $\mathrm{TiO}_{2}$-based photocatalysts need to be improved markedly.

To narrow the intrinsic band gap and extend the response region of $\mathrm{TiO}_{2}$ to the Vis or IR light, large number of elements are doped to obtain colourful materials [10-15, 17, 18]. However, these strategies show little improvement in aspect of the catalytic efficiency owing to the enhanced recombination rate of photogenerated electrons and holes, and low carrier concentrations. Although $\mathrm{Ti}^{3+}$-self doped $\mathrm{TiO}_{2}$ can enhance the solar energy absorption, numerous bulk defects are unfavourable for charge transfer [19]. Surface defects like oxygen vacancies $\left(V_{o}\right)$ over semiconductors are proved to efficiently facilitate the catalytic performance [20]. Moreover, an appropriate amount of the surface defects can induce the generation of intermediate states of $\mathrm{Ti}^{3+}$ species which serve as lightgenerated carrier sinks for retarding charge recombination [21]. During the search for a readily available and applicable strategy for the preparation of full-spectrum solar responsive $\mathrm{TiO}_{2}$-based materials for energy transformation and chemical conversion, we have recently demonstrated that several methods including $\mathrm{H}_{2}$-plasma, metal reduction, and element doping were widely used for the synthesis of various black, red, gray, and blue titania [22-26]. Compared to pristine $\mathrm{TiO}_{2}$, these colored samples with unique crystalline-core/amorphous-shell structure and large amount of surface $\mathrm{V}_{\mathrm{o}}$ species can enhance the separation and transportation of photoinduced $\mathrm{e}^{-}-\mathrm{h}^{+}$pairs and thus the carrier concentrations. Thus, the extraordinary structure and physicochemical properties of colored $\mathrm{TiO}_{2}$ endow its superior microwave and terahertz absorption [27-32], special solar light absorption and excellent photocatalysis performance.

Herein, we report that the conductive black titania (BT) fabricated via Al-reduction method shows exceptional photocatalytic efficiency for the degradation of organic pollutants (e.g., toxic toluene and ethyl acetate) under solar irradiation and mild reaction conditions. The key to the successful use of the designed photocatalysts is the unique crystalline-core/amorphous-shell structure and numerous surface $\mathrm{V}_{\mathrm{o}}$ showing narrow band gap, high carrier concentration, prominent electron mobility, and excellent separation and transportation of photoinduced $\mathrm{e}^{-}-\mathrm{h}^{+}$pairs. These special physicochemical properties of series BT samples favor efficient adsorption, chemical activation, and photocatalytic oxidation transformation of organic pollutant molecule. The engineered BT-500 catalyst achieves superior photocatalytic degradation rates for toluene and ethyl acetate under full-spectrum solar and even visible light irradiation, about three-fold higher than those of the pristine $\mathrm{TiO}_{2}$. In addition, the catalyst gives excellent photostability with high degradation efficiency of $93 \%$ for the 6 th reuse. The present findings are promising not only for offering an effective photocatalyst system for degradation of toxic organic pollutants to environmentally friendly products, but also for obtaining novel insights into colored titania catalyzed reactions.

\section{Experimental}

\subsection{Chemicals and Materials}

$\mathrm{TiO}_{2}$ (P25 with $80 \%$ anatase phase and $20 \%$ rutile phase, $99.8 \mathrm{wt} \%)$ was obtained from Evonik. Al powders (99.98 wt \%) and Pt wire (99.95 wt\%) were supplied by Alfa Aesar. Toluene (99.5 wt\%), ethyl acetate $(99.5 \mathrm{wt} \%)$, and $\mathrm{NaOH}$ (98 wt\%) were supplied by Sinopharm Chemical Reagent Co., Ltd (SCRC). All the chemicals were used without further purification.

\subsection{Catalyst Preparation}

The preparation of BT samples were conducted in a twozone furnace (Fig. S1) [25], in which $\mathrm{TiO}_{2}$ and $\mathrm{Al}$ powders 
were separately placed at a pressure of $<0.5 \mathrm{~Pa}$. Later, Al was heated to $800{ }^{\circ} \mathrm{C}$, and the $\mathrm{TiO}_{2}$ was heated to $300-500{ }^{\circ} \mathrm{C}$ for $6 \mathrm{~h}$. After the annealing and pressure release, the BT samples were carefully collected.

\subsection{Catalyst Characterization}

Brunauer-Emmett-Teller (BET) specific surface areas of BT catalysts were determined by $\mathrm{N}_{2}$ adsorption-desorption at $77 \mathrm{~K}$, using a Micromeritics TriStar 3000 equipment. Sample degassing was carried out at $300{ }^{\circ} \mathrm{C}$ prior to acquiring the adsorption isotherm. Al content was measured by inductively coupled plasma-atomic emission spectroscopy (ICP-AES) using iCAP 6300 spectrometer. X-ray diffraction (XRD) information of the samples was carried out on a German Bruker D8 Advance X-ray diffractometer using nickel filtered $\mathrm{Cu} \mathrm{K} \alpha$ radiation at $40 \mathrm{kV}$ and $40 \mathrm{~mA}$. X-ray photoelectron spectroscopy (XPS) spectra were recorded with a Perkin Elmer PHI 5000C system equipped with a hemispherical electron energy analyzer. The spectrometer was operated at $15 \mathrm{kV}$ and $20 \mathrm{~mA}$, and a magnesium anode $(\mathrm{Mg} \mathrm{K} \alpha, \mathrm{h} \nu=1253.6 \mathrm{eV})$ was used. A JEOL 2011 microscope operating at $200 \mathrm{kV}$ equipped with an EDX unit $(\mathrm{Si}(\mathrm{Li})$ detector) was used for the transmission electron microscopy (TEM) investigations. A JEM 2100F electron microscope operating at $200 \mathrm{kV}$ equipped with an EDX unit ( $\mathrm{Si}(\mathrm{Li})$ detector) was used for the high-resolution (HR)TEM investigations. The samples for electron microscopy were prepared by dispersing the powder in ethanol and applying a drop of very dilute suspension on carbon-coated grids. UV-VIS-NIR diffuse reflectance (DR) measurement of the solids were recorded at room temperature on a Hitachi U4100 Spectrometer equipped with an integrating sphere and using $\mathrm{BaSO}_{4}$ as reference. The solar absorption of $\mathrm{TiO}_{2}$ samples was attained by the following Eq. (1) [23]:

$A=\frac{\int(1-T) \cdot S \cdot d \lambda}{\int S \cdot d \lambda}$

where $A$ is the solar absorption, $T$ is the reflectance of the sample, $S$ is the solar spectral irradiance $\left(\mathrm{W} \mathrm{m}^{-2} \mathrm{~nm}^{-1}\right), \lambda$ is the wavelength $(\mathrm{nm})$, and the (1-T) $S$ represents the sample absorption of solar spectral irradiance. Photothermic effect of the cool-pressed $\mathrm{TiO}_{2}$ and BT-500 powders were investigated under the irradiation of an AM 1.5G Xe lamp solar light simulator for different times. Raman spectra were collected in a thermal dispersive spectrometer using a $10 \mathrm{~mW}$ laser with an excitation wavelength of $532 \mathrm{~nm}$. Electron paramagnetic resonance (EPR) spectra were investigated using a Bruker EMX-8 spectrometer at $9.44 \mathrm{GHz}$ and $300 \mathrm{~K}$. Resistance measurement was conducted using standard four electrode method performed on physical property measurement system (PPMS) (DynaCool-9, Quantum Design Inc.) at room temperature. The carrier concentration was determined using Hall-effect measurement method at the magnetic field ranged from $-2 \mathrm{~T}$ to $2 \mathrm{~T}$ at room temperature. To characterize the transient photocurrent responses of the samples, a conventional three electrode system was used to conduct electrochemical measurements with an electrochemical workstation (CHI660B, CH Instruments). Pristine $\mathrm{TiO}_{2}$ films were prepared on FTO glass substrate by a spin-coating method. These films were first annealed at $500{ }^{\circ} \mathrm{C}$ for $1 \mathrm{~h}$ in air and then treated by $\mathrm{Al}$ reduction. The sample-coated FTO glasses were used directly as the working electrode, with a Pt wire and an $\mathrm{Ag} / \mathrm{AgCl}$ ( $\mathrm{KCl}$ saturated) electrode as counter and reference electrodes respectively in $1 \mathrm{M} \mathrm{NaOH}$ aqueous solution ( $\mathrm{pH}$ 13.6). A set of transient photocurrent responses were recorded in dark and under irradiation. A $150 \mathrm{~W}$ Xe lamp was used as the light source to simulate the sunlight irradiation. The light intensity was measured by a calibrated Si photodiode. Photoluminescence (PL) was excited at $320 \mathrm{~nm}$ using a fluorescence spectrophotometer of MODEL Fluoro Max-3 (Horiba) at room temperature.

\subsection{Adsorption Measurement}

Adsorption measurement before catalysis was carried out on the conditions of dark and $25{ }^{\circ} \mathrm{C}$. The initial concentration of aqueous toluene or ethyl acetate solution was $20 \mathrm{mg} \mathrm{L}^{-1}$. $50 \mathrm{mg}$ sample was added to $100 \mathrm{~mL}$ solution under stirring. At given time intervals, the selected solution (ca. $1 \mathrm{~mL}$ ) was analyzed using a gas chromatograph Agilent 7820A equipped with a HP-5 capillary column connected to a flame ionization detector.

\subsection{Photocatalytic Degradation Measurement}

Photocatalytic degradation was conducted by the decomposition of toluene or ethyl acetate in an aqueous solution with an initial concentration of $50 \mathrm{mg} \mathrm{L}^{-1} .50 \mathrm{mg}$ catalyst was added to $100 \mathrm{~mL}$ solution. A $300 \mathrm{~W}$ Xe lamp (Aulight CEL-HX, Beijing) was used as a light source for photocatalytic reaction. At given time intervals, the selected solution $(1 \mathrm{~mL})$ was analyzed using a gas chromatograph Agilent 7820A equipped with a HP-5 capillary column connected to a flame ionization detector.

Photostability test of BT-500 was carried out on solar light irradiation and $3 \mathrm{~h}$ in each run. After each reaction, the centrifuged catalysts from parallel tests were collected and washed with distilled water several times, followed by air-drying at $100{ }^{\circ} \mathrm{C}$ overnight. 


\section{Results and Discussion}

\subsection{Structural Characterization and Physicochemical Properties}

To examine the influence of $\mathrm{V}_{\mathrm{o}}$ and the amorphous shell on the photocatalytic degradation activity of BT, the pristine $\mathrm{TiO}_{2}$ treatment temperature is mainly focused on $300-500{ }^{\circ} \mathrm{C}$ in this study (Fig. S1). Thermodynamic analysis demonstrates that Al-reduction temperature of $800{ }^{\circ} \mathrm{C}$ is sufficient for the generation of melting aluminum and aluminium oxides and thus the $\mathrm{O}$-defective $\mathrm{TiO}_{2-\mathrm{x}}$ thin shell over crystalline $\mathrm{TiO}_{2}$ core. Note that the higher temperature of $600{ }^{\circ} \mathrm{C}$ can lead to the again crystallization of amorphous shell and the significant decrease of surface $\mathrm{V}_{\mathrm{o}}$ amount [23]. The obtained catalysts constituting of $\mathrm{TiO}_{2} @ \mathrm{TiO}_{2-\mathrm{x}}$ are denoted as BT-300, BT-400, and 500BT at the treatment temperature of 300,400 , and $500{ }^{\circ} \mathrm{C}$, respectively.

BT samples show no remarkable change in BET surface area and pore size properties relative to the pristine $\mathrm{TiO}_{2}$ (Fig. S3 and Table S1), which are consistent with the previous results [23]. It should be emphasized that there is no $\mathrm{Al}$ element in the final $\mathrm{BT}$ samples, as proved by XPS and ICP-AES analysis in Fig. $\mathrm{S} 4$. $\mathrm{TiO}_{2}$ powder was converted from white to gray of BT-300 and darkblue of BT-400 and black of BT-500 by increasing the reduction temperature (left column in Fig. 1), suggesting that the enhanced Vis-light absorption for dark color samples [33]. TEM images in Figs. S5 and 1b show that the pristine $\mathrm{TiO}_{2}$ is completely crystalline, displaying welldefined lattice fringes and well-ordered crystal domains of around $25 \mathrm{~nm}$. Compared with crystallized $\mathrm{TiO}_{2}$ (Fig. 1b), BT samples present a typical core-shell structure, as the HRTEM images depicted in Figs. 1d, f, and h, in which the core is remained the crystallized $\mathrm{TiO}_{2}$ with anatase (101) plane whereas the outer shell is amorphous $\mathrm{TiO}_{2-\mathrm{x}}$. Note that the distance between the adjacent lattice planes for these samples is remained ca. $3.5 \AA$. These results suggest that the Al-reduction process only affects the surface structure of pristine material. The thickness of the amorphous layer increases with the reduction temperature; that is, ca. $1 \mathrm{~nm}$ for BT-300 to $1-2 \mathrm{~nm}$ for BT- 400 to $2-3 \mathrm{~nm}$ for BT-500 (right column in Fig. 1). It was demonstrated that the disordered structure in metal oxides is favorable for the generation of surface defects and the presence of the crystallized-amorphous heterojunction is beneficial to the separation and transportation of photoinduced $\mathrm{e}^{-}-\mathrm{h}^{+}$ pairs [22-26].

XRD patterns in Fig. 2 reveal a negligible phase transition, in which BT samples remain high crystallinity with the co-existence of dominant anatase phase and rutile $\mathrm{TiO}_{2}$
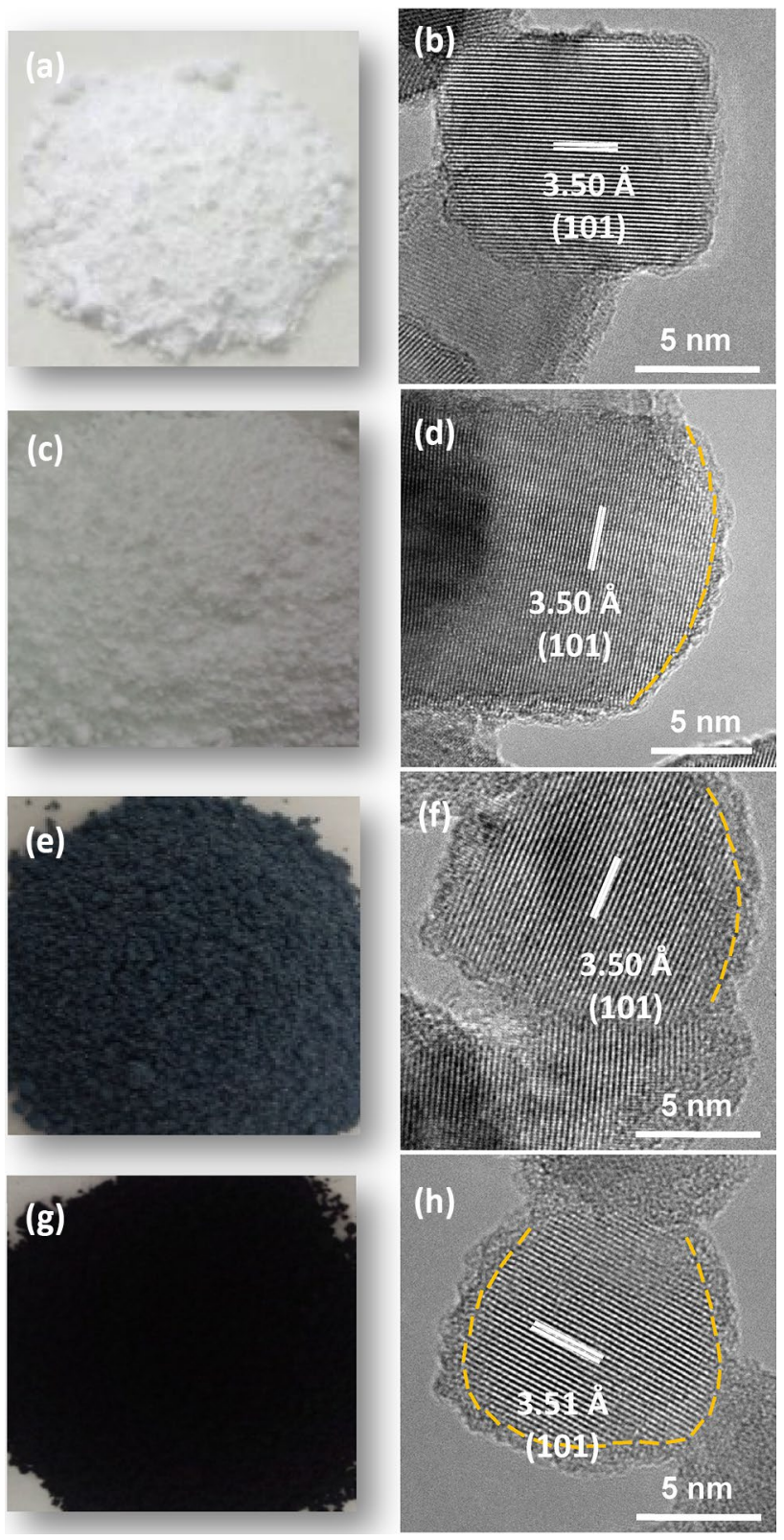

Fig. 1 Photographs and HRTEM images of $\mathbf{a}, \mathbf{b} \mathrm{TiO}_{2}, \mathbf{c}, \mathbf{d} \mathrm{BT}-300, \mathbf{e}$, f BT- 400 , and $\mathbf{g}$, h BT-500 samples

after Al-reduction treatment. The crystallite size of BT samples was estimated using Scherrer equation to be about $25 \mathrm{~nm}$, which is similar to the pristine $\mathrm{TiO}_{2}$. As the unique crystalline-core/amorphous-shell structure was formed, the colored titania was generated. For BT-500 catalyst, the typical core-shell structure also results in a narrow band gap of ca. $2.8 \mathrm{eV}$ (Fig. S6), which is much lower than that of pristine $\mathrm{TiO}_{2}$. BT samples significantly enhance the absorption of Vis and even near IR light, as illustrated in Fig. 3. The pristine $\mathrm{TiO}_{2}$ only shows response on ultraviolet (UV) light. The absorption spectra demonstrate the increased enhancement with the Al-reduction temperature 


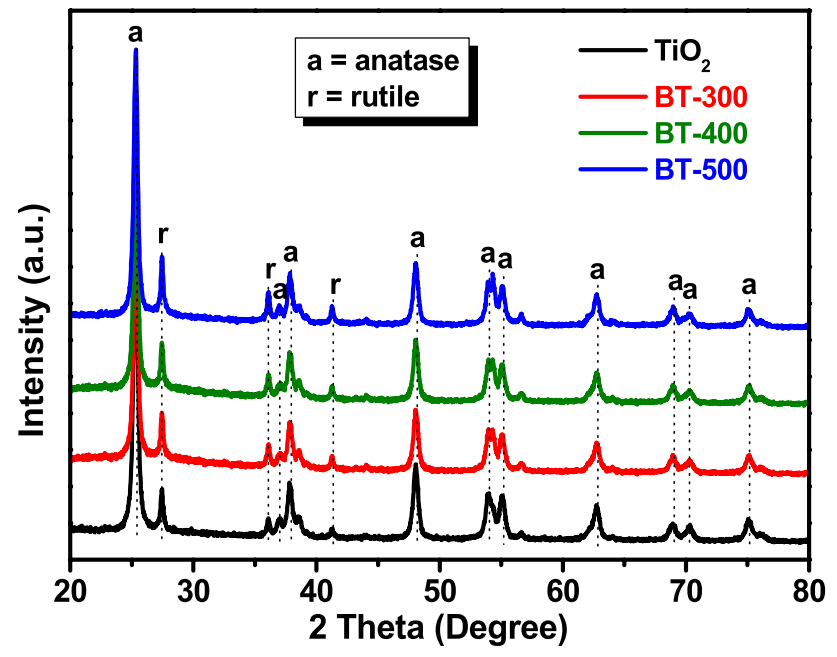

Fig. 2 XRD patterns of $\mathrm{TiO}_{2}$ and $\mathrm{BT}$ samples

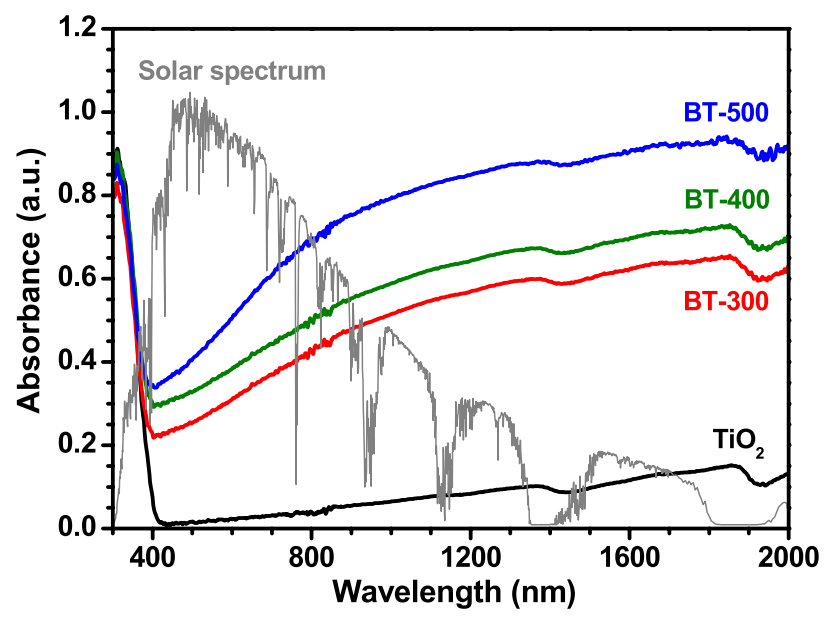

Fig. 3 UV-VIS-NIR Diffuse reflectance spectra of $\mathrm{TiO}_{2}$ and BT samples

increasing. For example, BT-500 possesses far larger solar spectrum absorption (ca. 71\% of solar spectral irradiance) than those of BT-400 ( 60\%) and BT-300 ( 53\%).

The enhanced solar absorption of BT materials was further investigated by the photothermic effect under the irradiation of an AM 1.5G Xe lamp solar light simulator. The investigated disks were cool-pressed from pristine $\mathrm{TiO}_{2}$ and BT-500 powders. As depicted in Fig. 4, after being irradiated for $60 \mathrm{~s}$, the temperature of the BT-500 disk increased to $38^{\circ} \mathrm{C}$, which was much higher than the $30{ }^{\circ} \mathrm{C}$ for the pristine $\mathrm{TiO}_{2}$ disk. The accelerated heating rate of the BT-500 disk is ascribed to larger solar absorption illustrated by the absorption spectrum in Fig. 3, resulting in more electron excitation and transition and the enhanced heat emission. Thus, the conductive black titania materials can be widely applied as a solar absorber medium for solar thermal collection.

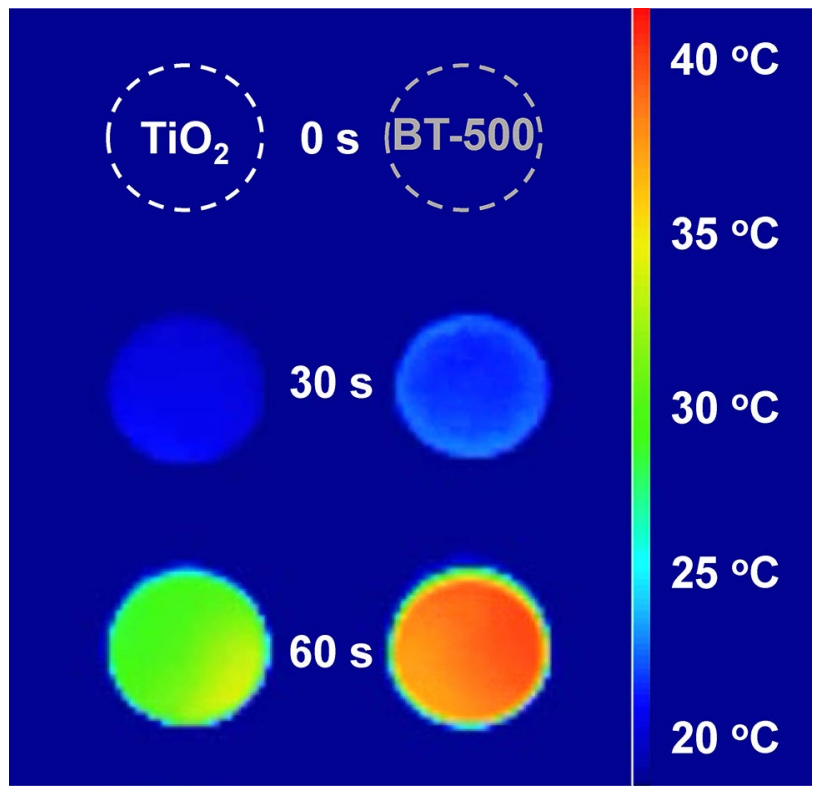

Fig. 4 Thermal image map of cool-pressed disks of $\mathrm{TiO}_{2}$ and BT-500 samples under solar simulator irradiation for different times

The surface amorphous layer and $\mathrm{V}_{\mathrm{o}}\left(\right.$ or $\left.\mathrm{Ti}^{3+}\right)$ can be proved by Raman spectroscopy. The strongest signal of $\mathrm{E}_{g}$ mode at $145 \mathrm{~cm}^{-1}$ depicted in Fig. 5a is assigned to the external vibration of Ti-O bond [25]. This demonstrates a visible blue-shift (from 145 of $\mathrm{TiO}_{2}$ to $148 \mathrm{~cm}^{-1}$ of BT-300 to $154 \mathrm{~cm}^{-1}$ of BT-500) accompanied the slight peak broadening along with the Al-reduction treatment at elevated temperatures. The latter is probably due to the existence of numerous surface defects $\left(\mathrm{Ti}^{3+}\right.$ or $\left.\mathrm{V}_{\mathrm{o}}\right)$ in amorphous $\mathrm{TiO}_{2-\mathrm{x}}$ layer [34]. XPS results illustrated in Fig. 5b show a slight shift to lower energy for Ti2p peak of BT samples, which are consistent well with the Raman data (Fig. 5a). There is no appearance of $\mathrm{Ti}^{3+}$ species in pristine $\mathrm{TiO}_{2}$, while obvious signal of $\mathrm{Ti}^{3+}$ can be observed in BT samples (Fig. 5b) and the proportion of $\mathrm{Ti}^{3+}$ in total Ti species increases with Alreduction temperature (Table $\mathrm{S} 2$ ). EPR as a highly sensitive technique is widely applied to probe paramagnetic species containing the unpaired electrons in $\mathrm{V}_{\mathrm{o}}$ and/or $\mathrm{Ti}^{3+}{ }^{322-26]}$. There is a strong EPR signal with the $g$-value of 2.003 for BT catalysts, as depicted in Fig. 5c. This is ascribed to the unpaired electrons [35], indicative of the presence of $\mathrm{V}_{\mathrm{o}}$ or $\mathrm{Ti}^{3+}$ species in surface amorphous $\mathrm{TiO}_{2-x}$ layer. Moreover, the EPR intensity increases gradually with the Al-reduction temperature. In order to reveal the behavior of photogenerated holes and electrons, PL emission tests were carried out. Fig. S7 shows the PL spectra of pristine $\mathrm{TiO}_{2}$ and BT samples in the wavelength region of $350-650 \mathrm{~nm}$. The intensities of BT catalysts are much weaker than that of pristine $\mathrm{TiO}_{2}$, indicative of a much lower recombination rate of the holes and electrons irradiated by UV light source for BT 

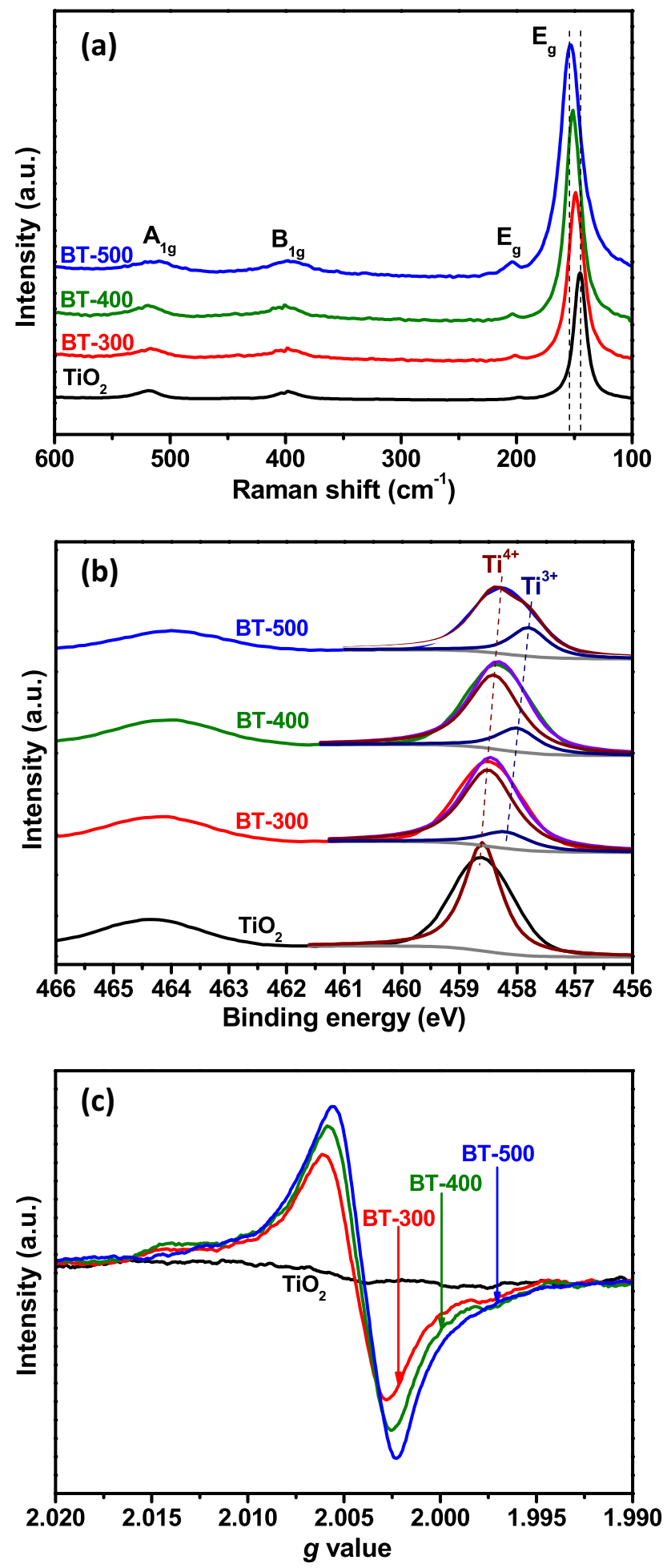

Fig. 5 a Raman, b Ti2p XPS, and c EPR data of $\mathrm{TiO}_{2}$ and BT samples

samples [22-26]. BT-500 shows the lowest PL peak intensity. The unique properties of the crystalline-core/amorphous-shell structured BT with abundant $\mathrm{V}_{\mathrm{o}}$ or $\mathrm{Ti}^{3+}$ species can significantly reduce the recombination rate of $\mathrm{e}^{-}-\mathrm{h}^{+}$ pairs. Thus, efficiently promoting the separation and transportation of $\mathrm{e}^{-}-\mathrm{h}^{+}$and the migration/relaxation of charge carriers within the BT matrix.

It was demonstrated that the $\mathrm{Ti}^{3+}$ species can generate $0.7-1.0 \mathrm{eV}$ below the conduction band minimum (CBM) and just below the $\mathrm{CB}$ tail in the electronic structure of $\mathrm{BT}$ sample [23]. Mild thermal treatment conditions or even photons with relatively low energy can also induce the $\mathrm{Ti}^{3+}$ $3 \mathrm{~d}^{1}$ electron to band tail or upper energy levels (Fig. S8). This shows a positive relationship to the highly enhanced electron concentration. In our previous study [23], the electron concentrations of BT-400 and BT-500 were recorded to be $8.87 \times 10^{17} \mathrm{~cm}^{-3}$ and $7.41 \times 10^{18} \mathrm{~cm}^{-3}$, respectively, according to the Hall measurements. Note that the electron concentrations of BT samples are about two orders of magnitude higher than that of $\mathrm{TiO}_{2}$. Meanwhile, the transport and accumulation of photogenerated charges as well as the heat produced in semiconductors (e.g., titania and colored titania) are essential in photocatalytic reactions [36-38]. Moreover, the current values are almost zero in dark while the photocurrent rapidly rises to a steady-state value with illumination. The observed steady-state photocurrent of BT-500 is $1.3 \mathrm{~mA} \mathrm{~cm}^{-2}$ (Fig. S9), which is approximately eight times larger than that of pristine $\mathrm{TiO}_{2}\left(0.17 \mathrm{~mA} \mathrm{~cm}^{-2}\right)$, indicative of a much higher concentration of photo-induced carriers in BT samples due to the enhanced light-absorption as well as the effective separation and rapid transportation of light-generated carriers. These prominent properties can fulfill the potential requirement for photocatalytic degradation of organic pollutants over BT samples.

\subsection{Photocatalytic Degradation of Organic Pollutants}

The excellent photocatalytic performance of the BT samples was demonstrated by the degradation of toluene, as illustrated in Fig. 6. Note that the transformations did not occur at all without the use of BT catalysts. BT catalysts exhibit considerable full solar light and even Vis light activity, whereas the pristine $\mathrm{TiO}_{2}$ gives low activity under the same reaction conditions (Fig. 6). The catalytic efficiency of BT samples increases with the Al-reduction temperature, and BT-500 is the best. This is due to the more enhanced light absorption and lager number of surface $\mathrm{V}_{\mathrm{o}}$ for BT-500 than other samples. It should be emphasized that BT-500 needs only 3 and $5 \mathrm{~h}$ for the degradation efficiency of more than 95\% for toluene under full solar light and Vis light irradiation, respectively (Fig. 6). The degradation rates are about three-fold higher than those of the pristine $\mathrm{TiO}_{2}$. Moreover, BT samples exhibit significantly promoted activities for ethyl acetate degradation under full-spectrum solar irradiation with the similar tendency of toluene degradation, as 


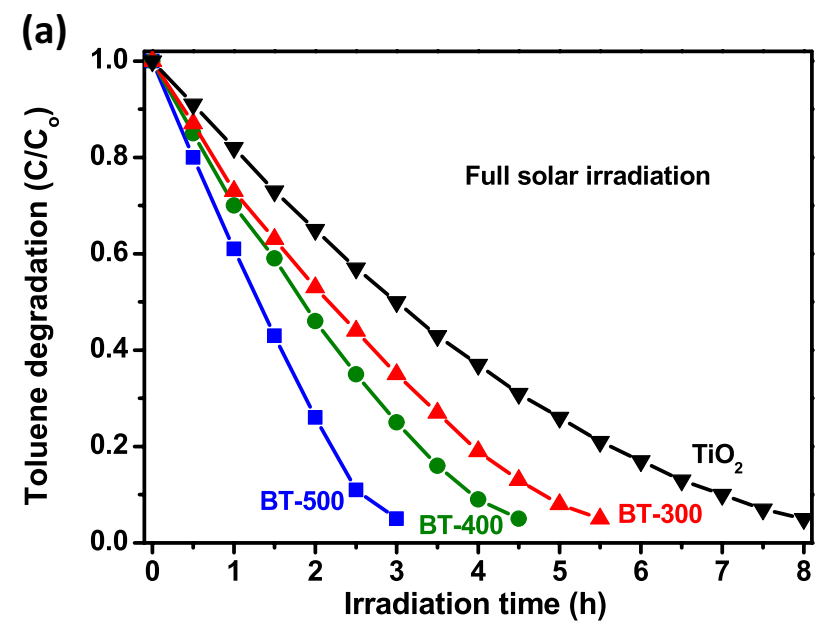

(b)

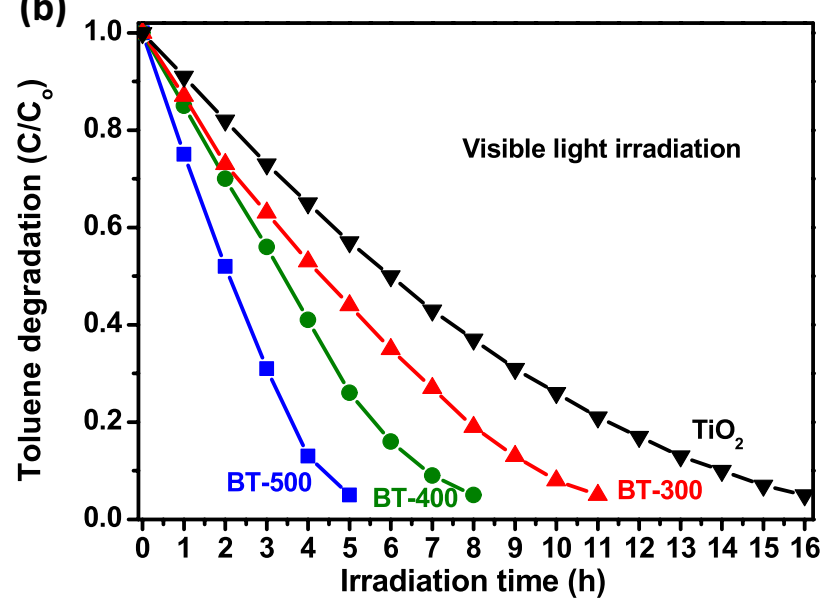

Fig. 6 Photocatalytic activities of $\mathrm{TiO}_{2}$ and BT samples for toluene degradation under $\mathbf{a}$ full solar and $\mathbf{b}$ visible light irradiation

the results depicted in Fig. 7. The degradation rate of ethyl acetate (BT-500 needs $6 \mathrm{~h}$ for the degradation efficiency of ca. $90 \%$ under full solar light irradiation) is much lower than that of toluene due to the complicated molecular structure of the former. These results demonstrate that BT catalysts, especially BT-500 with numerous surface $V_{o}$, which possesses a narrowed band gap and prominent properties on solar and even Vis light absorption, achieves a superior efficiency for photoinduced degradation of organic pollutants.

Compared to the photocatalytic degradation of toluene and ethyl acetate under light irradiation, BT samples give low efficiency on adsorption capacity (Fig. S10). These results show that the physical adsorption process is easily saturated in limited time and the adsorbate is hard to be desorbed or converted downstream without external driving force [39-42]. As an important step of overall catalytic process, the adsorption with high efficiency is usually favorable for the catalytic transformation in practical conditions [43-45]. BT-500 sample with the largest amount of surface

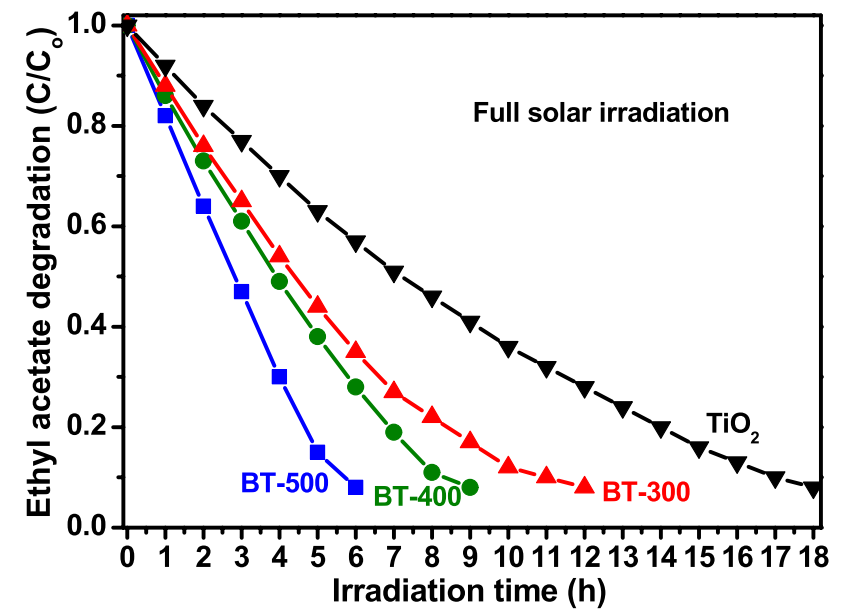

Fig. 7 Photocatalytic activities of $\mathrm{TiO}_{2}$ and BT samples for ethyl acetate degradation under full solar light irradiation

$\mathrm{V}_{\mathrm{o}}$ shows the best adsorption efficiency for toluene and ethyl acetate (Fig. S10), thus displays the highest photocatalytic degradation for both organic pollutants under solar or Vis light irradiation (Figs. 6 and 7).

Having established that BT-500 catalyzed photoinduced degradation of toluene and ethyl acetate as a reliable and efficient catalysis system for oxidation removal of organic pollutants, we further studied the reusability of the photocatalyst under solar light irradiation. As illustrated in Fig. 8, BT-500 shows excellent stability for the degradation of toluene under full-spectrum solar light irradiation. This sample can be reused up to six cycles without remarkable loss in activity. The toluene degradation efficiency remains at a high level of $93 \%$ for the 6th reuse. Compared with fresh BT-500, there is no significant

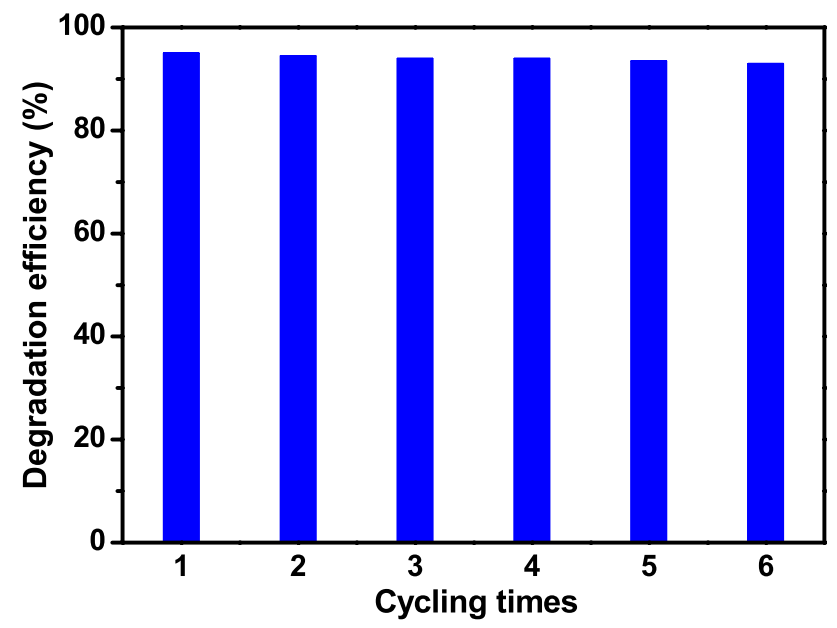

Fig. 8 Cycling tests of BT-500 for toluene degradation under full solar light irradiation 
change on the aspects of the crystalline- $\mathrm{TiO}_{2}$-core/amorphous- $\mathrm{TiO}_{2-\mathrm{x}}$-shell structure, black feature, abundant $\mathrm{V}_{\mathrm{o}}$, and prominent separation and transportation of photoinduced $\mathrm{e}^{-}-\mathrm{h}^{+}$pairs of the used catalyst (Figs. 9, S11, and S12). It should be noted that the used BT-500 still shows distinct EPR signal corresponding to $\mathrm{V}_{\mathrm{o}}$ or $\mathrm{Ti}^{3+}$ after photocatalytic degradation reaction for overall $18 \mathrm{~h}$ (Fig. 9b). These results further demonstrate the effectiveness of BT catalysts, especially BT-500 for full solar light excited photocatalytic degradation of organic pollutants under viable conditions.
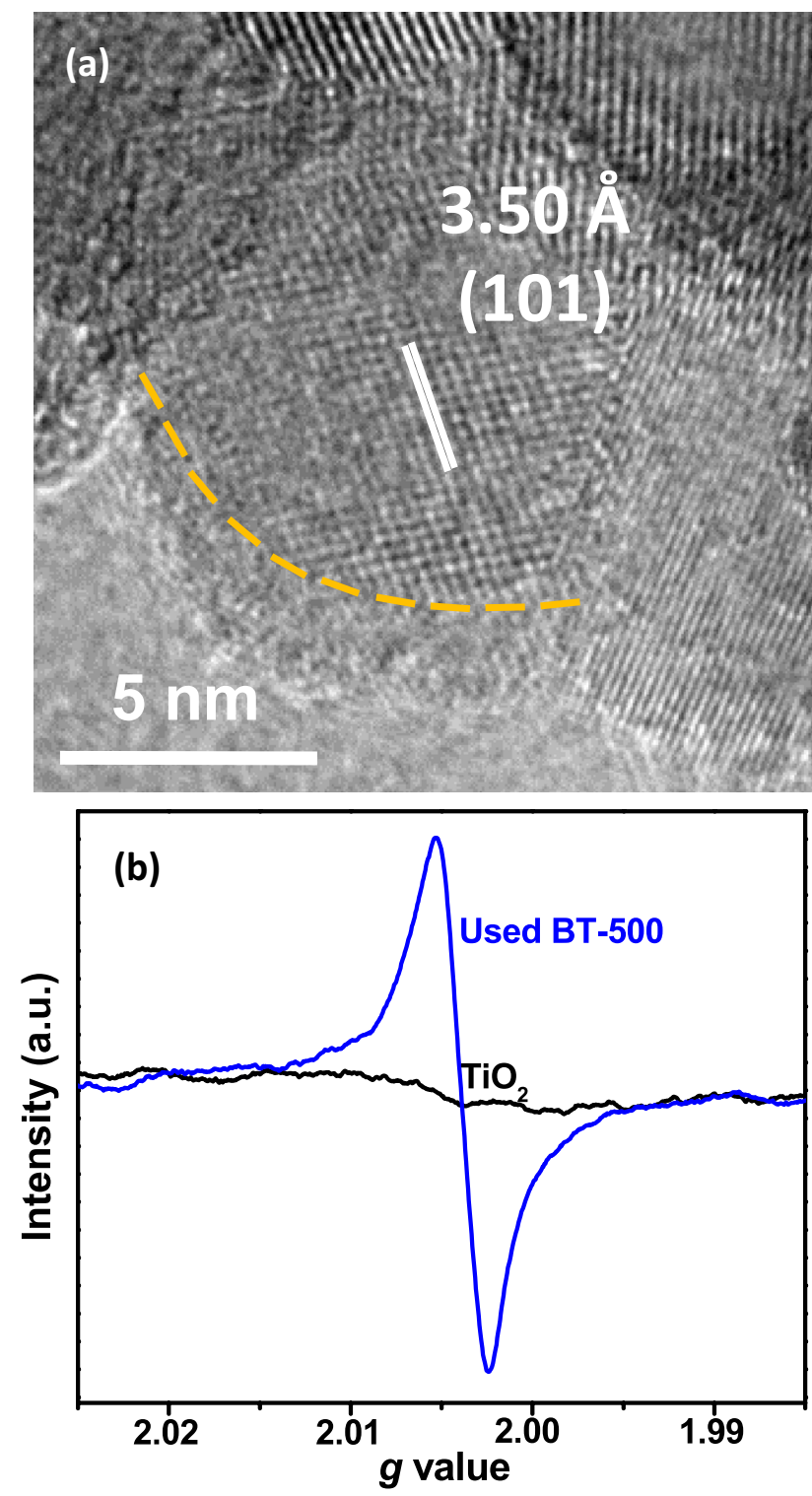

Fig. 9 a HRTEM image and b EPR spectrum of the used BT-500 sample

\section{Conclusions}

Efficient conductive black titania nanomaterisls with typical crystalline- $\mathrm{TiO}_{2}$-core/amorphous- $\mathrm{TiO}_{2-\mathrm{x}}$-shell structure were successfully fabricated by using two-zone Al-reduction strategy. Series BT catalysts were tested for the photocatalytic degradation of toxic organic pollutants (toluene and ethyl acetate) to environmentally friendly products under full solar or Vis light irradiation and mild reaction conditions. The unique core-shell structure and numerous surface $\mathrm{V}_{\mathrm{o}}$ or $\mathrm{Ti}^{3+}$ species in the amorphous layer accompanying prominent physicochemical properties of narrow band gap, high carrier concentration, high electron mobility, and excellent separation and transportation of photoinduced $\mathrm{e}^{-}-\mathrm{h}^{+}$ pairs resulted in exceptional photocatalytic efficiency. The engineered BT-500 catalyst achieved superior photocatalytic degradation rates for toluene and ethyl acetate under fullspectrum solar and even Vis light irradiation, and an excellent photostability with high degradation efficiency of $93 \%$ for the 6 th reuse. These findings are promising not only for offering an effective photocatalyst system for toxic organic pollutants degradation, but also for obtaining novel insights into colored titania catalyzed reactions.

Acknowledgements This work was financially supported by the National Key Research and Development Program of China (2016YFB0901600), the NSF of China (21872166), and the Key Research Program of Chinese Academy of Sciences (QYZDJ-SSWJSC013 and KGZD-EW-T06).

\section{Compliance with Ethical Standards}

Conflict of interest The authors declare no competing financial interest.

Open Access This article is distributed under the terms of the Creative Commons Attribution 4.0 International License (http://creativecommons.org/licenses/by/4.0/), which permits unrestricted use, distribution, and reproduction in any medium, provided you give appropriate credit to the original author(s) and the source, provide a link to the Creative Commons license, and indicate if changes were made.

\section{References}

1. Liotta LF (2010) Appl Catal B 100:403-412

2. Huang Z, Gu X, Cao Q, Hu P, Hao J, Li J, Tang X (2012) Angew Chem Int Ed 51:4198-4203

3. Pelaez M, Nolan NT, Pillai SC, Seery MK, Falaras P, Kontos AG, Dunlop PSM, Hamilton JWJ, Byrne JA, O’Shea K, Entezari MH, Dionysiou DD (2012) Appl Catal B 125:331-349

4. Quici N, Vera ML, Choi H, Puma GL, Dionysiou DD, Litter MI, Destaillats H (2010) Appl Catal B 95:312-319

5. Maira AJ, Coronado JM, Augugliaro V, Yeung KL, Conesa JC, Soria J (2001) J Catal 202:413-420 
6. Wang CC, Li JR, Lv XL, Zhang YQ, Guo G (2014) Energy Environ Sci 7:2831-2867

7. Zhao W, Ma W, Chen C, Zhao J, Shuai Z (2004) J Am Chem Soc 126:4782-4783

8. Di J, Xia J, Ge Y, Li H, Ji H, Xu H, Zhang Q, Li H, Li M (2015) Appl Catal B 168-169:51-61

9. Xu D, Cheng B, Cao S, Yu J (2015) Appl Catal B 164:380-388

10. Chen X, Liu L, Yu PY, Mao SS (2011) Science 331:746-750

11. Liu L, Chen X (2014) Chem Rev 114:9890-9918

12. Chen X, Liu L, Huang F (2015) Chem Soc Rev 44:1861-1885

13. Liu X, Zhu G, Wang X, Yuan X, Lin T, Huang F (2016) Adv Energy Mater 17:1600452

14. Chang W, Sun C, Pang X, Sheng H, Li Y, Ji H, Song W, Chen C, Ma W, Zhao J (2015) Angew Chem Int Ed 54:2052-2056

15. Liu L, Zhao H, Andino JM, Li Y (2012) ACS Catal 2:1817-1828

16. Habisreutinger SN, Schmidt-Mende L, Stolarczyk JK (2013) Angew Chem Int Ed 52:7372-7408

17. Schneider J, Matsuoka M, Takeuchi M, Zhang J, Horiuchi Y, Anpo M, Bahnemann DW (2014) Chem Rev 114:9919-9986

18. Liu G, Yang HG, Pan J, Yang YQ, Lu GQ, Cheng HM (2014) Chem Rev 114:9559-9612

19. Xin X, Xu T, Wang L, Wang C (2016) Sci Rep 6:23684

20. Lei F, Sun Y, Liu K, Gao S, Liang L, Pan B, Xie Y (2014) J Am Chem Soc 136:6826-6829

21. Li L, Yan J, Wang T, Zhao ZJ, Zhang J, Gong J, Guan N (2015) Nat Commun 6:5881

22. Wang Z, Yang C, Lin T, Yin H, Chen P, Wan D, Xu F, Huang F, Lin J, Xie X, Jiang M (2013) Adv Funct Mater 23:5444-5450

23. Wang Z, Yang C, Lin T, Yin H, Chen P, Wan D, Xu F, Huang F, Lin J, Xie X, Jiang M (2013) Energy Environ Sci 6:3007-3014

24. Yang C, Wang Z, Lin T, Yin H, Lü X, Wan D, Xu T, Zheng C, Lin J, Huang F, Xie X, Jiang M (2013) J Am Chem Soc 135:17831-17838

25. Yin G, Bi Q, Zhao W, Xu J, Lin T, Huang F (2017) ChemCatChem 9:4389-4396

26. Yin G, Huang X, Chen T, Zhao W, Bi Q, Xu J, Han Y, Huang F (2018) ACS Catal 8:1009-1017

27. Green MA, Xu J, Liu H, Zhao J, Li K, Liu L, Qin H, Zhu Y, Shen D, Chen X (2018) Mater Today Phys 4:64-69

28. Tian L, Xu J, Just M, Green M, Liu L, Chen X (2017) J Mater Chem C 5:4645-4653
29. Li K, Xu J, Yan X, Liu L, Chen X, Luo Y, He J, Shen DZ (2016) Appl Phys Lett 108:183102

30. Xia T, Cao Y, Oyler NA, Murowchick J, Liu L, Chen X (2015) ACS Appl Mater Interfaces 7:10407-10413

31. Xia T, Zhang C, Oyler NA, Chen X (2014) J Mater Res 29:2198-2210

32. Xia T, Zhang C, Oyler NA, Chen X (2013) Adv Mater 25:6905-6910

33. Hamad H, Bailón-García E, Maldonado-Hódar FJ, Pérez-Cadenas AF, Carrasco-Marín F, Morales-Torres S (2019) Appl Catal B 241:385-392

34. Shao GS, Wang FY, Ren TZ, Liu Y, Yuan ZY (2009) Appl Catal B 92:61-67

35. Zhou X, Häublein V, Liu N, Nguyen NT, Zolnhofer EM, Tsuchiya H, Killian MS, Meyer K, Frey L, Schmuki P (2016) Angew Chem Int Ed 55:3763-3767

36. Guan L, Chen X (2018) ACS Appl Energy Mater 1:4313-4320

37. Xia T, Li N, Zhang Y, Kruger MB, Murowchick J, Selloni A, Chen X (2013) ACS Appl Mater Interfaces 5:9883-9890

38. Zhang Y, Harris CX, Wallenmeyer P, Murowchick J, Chen X (2013) J Phys Chem C 117:24015-24022

39. Essawy NAE, Ali SM, Farag HA, Konsowa AH, Elnouby M, Hamad HA (2017) Ecotoxicol Environ Safety 145:57-68

40. Hamad H, El-Latif MA, Kashyout AE, Sadik W, Feteha M (2015) New J Chem 39:3116-3128

41. Fathy M, Hamad H, Kashyout AE (2016) RSC Adv 6:7310-7316

42. Hamad HA, Sadik WA, El-latif MMA, Kashyout AB, Feteha MY (2016) J Environ Sci 43:26-39

43. Hamad H, El-latif MA, Kashyout AE, Sadik W, Feteha M (2015) Process Safety Environ Prot 98:390-398

44. Hamad H, Bailón-García E, Morales-Torres S, Carrasco-Marín F, Pérez-Cadenas AF, Maldonado-Hódar FJ (2018) J Environ Chem Eng 6:5032-5041

45. Hamad H, Castelo-Quibén J, Morales-Torres S, Carrasco-Marín F, Pérez-Cadenas AF, Maldonado-Hódar FJ (2018) Mater 11:1766

Publisher's Note Springer Nature remains neutral with regard to jurisdictional claims in published maps and institutional affiliations. 